\title{
Oscillatory Control and the Effects of Actuation Frequency on a Wall-Mounted Hump
}

\author{
Jennifer A. Franck* and Tim Colonius ${ }^{\dagger}$ \\ Division of Engineering and Applied Sciences, California Institute of Technology, Pasadena, CA 91125
}

\begin{abstract}
A large eddy simulation (LES) is used to explore the effects of high frequency actuation on a wall-mounted hump geometry. Low frequency forcing at $F^{+} \sim O(1)$ has been shown to increase entrainment through regular shedding of large-scale structures. Using a LES technique previously validated on the baseline and controlled flow over the wall-mounted hump geometry, the effectiveness of high frequency actuation is explored and compared with previous investigations. It is found that the high frequency actuation does not produce distinct vortical structures at the actuation frequency. The resulting mean flow is relatively unaltered by the application of high frequency actuation, although the local flow surrounding the actuation location is slightly modified.
\end{abstract}

\section{Introduction}

Flow separation is almost always detrimental to aerodynamic performance by increasing drag, decreasing lift, or producing undesirable oscillations. Synthetic jets have been shown to increase the aerodynamic performance of naturally separating flows in the laboratory. Through zero net-mass flux oscillation, synthetic jets add momentum to the flow, modifying the natural instabilities of the separated shear layer. Periodic excitation is often characterized by the amount of momentum added to the flow, defined by the unsteady momentum coefficient, $\left\langle C_{\mu}\right\rangle$, and the actuation frequency, non-dimensionalized by the separation distance $X_{\text {sep }}$ and freestream velocity $U_{\infty}$, as

$$
F^{+}=\frac{f X_{s e p}}{U_{\infty}} .
$$

The effective range of actuation frequencies for periodic excitation has been explored in many experimental ${ }^{1,2}$ and computational ${ }^{3-5}$ investigations of separated flows. Actuation frequencies are generally divided into two regimes, one is on the order of the natural large-scale shedding frequency, or $F^{+} \sim O(1)$. At this frequency large-scale vortices are created which increase the entrainment rate and deflect the separated shear layer towards the surface. This frequency scales with the separation bubble length, $X_{\text {sep }}$, and creates structures approximately the same height as the separation region. The deflected flap experiments of Nishri and Wygnanski ${ }^{6}$ determined that the boundary layer reattached at the lowest $\left\langle C_{\mu}\right\rangle$ when forced at a reduced frequency of $F^{+} \sim 1$. Airfoil investigations have also increased lift in post-stall angles of attack for actuation frequencies $0.5<F^{+}<1.5,^{7}$ a range that corresponds to the natural shedding frequency of the separated shear layer. The addition of oscillatory control regularizes the natural shedding of the large scale vortices, which increases entrainment within the separated region, and can delay separation or promote an earlier reattachment. ${ }^{8}$

The second regime of higher actuation frequencies is not as clearly understood. In this paper, high frequency actuation is defined by frequencies approximately an order of magnitude larger than the natural shedding or global frequency of the separated flow. In a turbulent flow, these higher frequencies are associated with the natural Kelvin-Helmholtz instabilities of the separated shear layer. In the airfoil experiments of Amitay and Glezer, ${ }^{1}$ high frequency periodic excitation is more effective in improving the aerodynamic performance than actuation at lower frequencies. High frequency forcing increased the suction force immediately after leading edge actuation, and has lower levels of lift and drag fluctuations than equivalent

${ }^{*}$ Current affliation: Postdoctoral Scholar, Division of Engineering, Brown University, Member AIAA

${ }^{\dagger}$ Professor, Mechanical Engineering, Senior Member AIAA 
low frequency actuation. They also discovered that above a certain threshold $F^{+}>10$ all high frequencies exhibited the same averaged pressure forces, and are decoupled from the natural shedding instabilities of the flow. Velocity profiles and instantaneous vorticity plots show no evidence of large scale structures or reverse flow close to the surface, indicating that the flow is completely attached and separation is prevented. The authors claim that the benefits of high frequency forcing are due to "virtual surface shaping" around the actuation location, or a modification to the average streamlines measuring 2-4 actuation wavelengths downstream. $^{2}$

Raju et al. ${ }^{4}$ looked at a stalled airfoil using two-dimensional direct numerical simulations (DNS) at $R e=44,000$, and noted the presence of multiple instabilities in the baseline flow. These can be attributed to Kelvin-Helmholtz instabilities of the shear layer at $S t_{s h} \approx 12$, a shedding frequency from the roll up of vortices in the separated region $S t_{s h} \approx 2$, and a low frequency in the wake of the airfoil $S t_{s h} \approx 1$. Forcing at frequencies close to the natural shedding instability are found to be most effective in reducing separation and increasing the lift-to-drag ratio. Contrary to the experiments by Amitay and Glezer, ${ }^{1}$ forcing at the shear layer frequency had an unfavorable effect of increasing the separated region. Dandois et al. ${ }^{5}$ also found an increase in separation bubble length when high frequency forcing is investigated in the LES of a curved backward-facing step. The low frequency increases entrainment and turbulent kinetic energy whereas the high frequency modifies the mean streamwise velocity profile stability and decreases the local kinetic energy.

In this investigation, the effect of actuation frequency is computationally investigated on a wall-mounted hump model. The wall-mounted hump was experimentally investigated by Seifert and Pack ${ }^{9}$ and the NASA Langley Research Center's CFD Validation of Synthetic Jets and Turbulent Separation Control workshop (LRCW) ${ }^{10,11}$ The model geometry is shown in figure 1, and features a highly convex region before the trailing edge, which initiates flow separation. The separated shear layer forms a turbulent and unsteady separation bubble over the trailing edge and eventually reattaches to the wall downstream of the model's chord length. Steady suction and low frequency oscillatory forcing have been shown to decrease the size of the separation bubble over the trailing edge of the hump. ${ }^{9-11}$

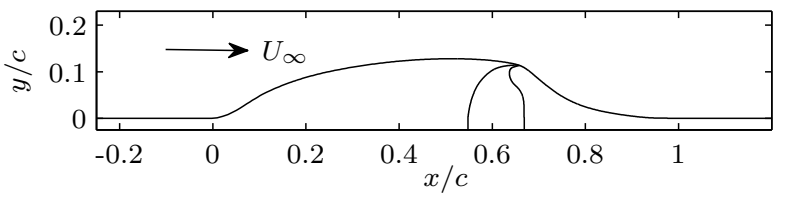

Figure 1. Wall-mounted hump geometry.

The wall-mounted hump has also been computationally investigated by a number of groups who simulated the baseline, steady suction and low frequency oscillatory test cases from LRCW, and are summarized by Rumsey. ${ }^{12}$ The current investigation uses a large eddy simulation (LES) ${ }^{13,14}$ that has been validated for the baseline and controlled wall-mounted hump test cases from LRCW, and the higher Mach number baseline flow from Seifert and Pack. ${ }^{15}$ The LES has been successful in predicting the unsteady vortex shedding of the low frequency actuated flow. In this paper, the LES is used to simulate the effects of high frequency actuation on the wall-mounted hump flow. The effectiveness and flow dynamics are examined, and the results are discussed and compared with the high frequency actuation performed in other investigations.

\section{Computational Methodology}

\section{II.A. Governing Equations}

The compressible large eddy simulation equations are derived by applying a spatial low-pass filter of width $\Delta$ to the compressible Navier-Stokes equations. The resulting mass, momentum and energy equations are

$$
\begin{aligned}
& \frac{\partial \bar{\rho}}{\partial t}+\frac{\partial}{\partial x_{j}} \bar{\rho} \tilde{u}_{j}=0 \\
& \frac{\partial}{\partial t} \bar{\rho} \tilde{u}_{i}+\frac{\partial}{\partial x_{j}}\left(\bar{\rho} \tilde{u}_{i} \tilde{u}_{j}-\tilde{\tau}_{j i}\right)+\frac{\partial \bar{p}}{\partial x_{i}}=\frac{\partial}{\partial x_{j}} \tau_{i j}^{s g s}
\end{aligned}
$$




$$
\frac{\partial}{\partial t} \bar{\rho} \tilde{E}+\frac{\partial}{\partial x_{j}}\left((\bar{\rho} \tilde{E}+\bar{p}) \tilde{u}_{j}+\tilde{q}_{j}-\tilde{\tau}_{j i} \tilde{u}_{i}\right)=\frac{\partial}{\partial x_{j}} q_{j}^{s g s}
$$

where $\tilde{f}$ represents the Favre-averaged low-pass filtered flow variable $f$. The quantities velocity and pressure are given by $u_{i}$ and $p$ respectively. The total energy is denoted by $E$ and is formulated by $E=e+0.5\left(u_{i} u_{i}\right)^{2}$, where $e$ is the internal energy per unit mass. The filtered stress tensor, $\tilde{\tau}_{i j}$, and heat flux vector, $\tilde{q}_{j}$, components are

$$
\begin{aligned}
& \tilde{\tau}_{i j}=\mu\left[\left(\frac{\partial \tilde{u}_{i}}{\partial x_{j}}+\frac{\partial \tilde{u}_{j}}{\partial x_{i}}\right)+\frac{2}{3} \frac{\partial \tilde{u}_{k}}{\partial x_{k}} \delta_{i j}\right] \\
& \tilde{q}_{j}=\frac{\mu}{\operatorname{Pr}} \frac{\partial \tilde{T}}{\partial x_{j}}
\end{aligned}
$$

where $\tilde{T}$ is the filtered temperature variable. The length scales are non-dimensionalized by the chord length, $c$, and the freestream values of density, $\rho_{\infty}$, and the speed of sound, $a_{\infty}$. The pressure is non-dimensionalized by $\rho a_{\infty}^{2}$. The dynamic viscosity is held constant, the Prandtl number is fixed at 0.7 , and the filtered ideal gas law is used as the equation of state, neglecting the subgrid transport terms that arise from filtering. The terms $\tau_{i j}^{s g s}$ represent the quantity $\bar{\rho}\left(\widetilde{u_{i} u_{j}}-\tilde{u}_{i} \tilde{u}_{j}\right)$, and $q_{i j}^{s g s}=\widetilde{T} u_{i}-\tilde{T} \tilde{u}_{i}$. These terms arise due to the filtering of products on the left-hand-side of the equations and cannot be calculated directly prompting the need for a subgrid scale (SGS) model. The LES uses an eddy-viscosity Smagorinsky formulation for compressible flows, ${ }^{16}$ and the SGS model terms are given by

$$
\begin{aligned}
\tau_{i j}^{s g s} & =C_{s} \Delta^{2} \bar{\rho}|\tilde{S}| \tilde{S}_{i j} \\
q_{j}^{s g s} & =C_{q} \Delta^{2} \bar{\rho}|\tilde{S}| \frac{\partial \tilde{T}}{\partial x_{j}}
\end{aligned}
$$

where $\Delta$ is the low pass filter width and $\tilde{S}_{i j}$ are the filtered rate of strain components. The filtered rate of strain is defined by

$$
\begin{aligned}
\tilde{S}_{i j} & =\frac{1}{2}\left(\frac{\partial \tilde{u}_{i}}{\partial x_{j}}+\frac{\partial \tilde{u}_{j}}{\partial x_{i}}\right) \\
|\tilde{S}| & =\left(2 \tilde{S}_{i j} \tilde{S}_{i j}\right)^{1 / 2}
\end{aligned}
$$

and the filter width $\Delta$, is calculated from the local grid spacings in the three coordinate directions, $\Delta=$ $\left(\Delta_{x} \Delta_{y} \Delta_{z}\right)^{1 / 3}$. A constant Smagorinsky model is implemented with the coefficients $C_{s}$ and $C_{q}$ set to 0.06. With the constant coefficient model, a van Driest damping function is used to decrease the characteristic length scale, $\Delta$, along the wall boundary using an empirical law of the wall formulation. ${ }^{17}$ The scaling function and related parameters are defined below.

$$
\begin{aligned}
& \Delta^{\prime}=\left\{1-\exp \left(x_{2}^{+} / A^{+}\right)\right\} \Delta \\
& x_{2}^{+}=x_{2} u_{\tau} / \nu, \quad A^{+}=25 \\
& \frac{\overline{u_{1}}}{u_{\tau}}=8.7 \cdot\left(y u_{\tau} / \nu\right)^{(1 / 7)}
\end{aligned}
$$

\section{II.B. Numerical Methods}

The governing equations are solved in a computational domain with the generalized coordinates $\xi=f(x, y)$ and $\eta=f(x, y)$ in the streamwise and wall normal directions. A conformal mapping from the computational domain $(\zeta=\xi+i \eta)$ to the physical domain $(z=x+i y)$ is calculated using the Schwartz-Christoffel Toolbox by Driscoll, ${ }^{18}$ capable of creating a conformal mapping from the equally spaced rectangular computational grid to an arbitrary physical grid defined by polygon vertices. The current hump geometry is defined by approximately 900 vertices denoted by the contour $\xi=0$. The smooth contour line just above the polygon boundary at $\xi=\epsilon$ is used as the lower boundary of the physical grid.

The wall parallel and wall normal derivatives are solved using a sixth-order explicit finite difference scheme with third-order boundary closure derived from summation by parts (SBP) operators based on diagonal norms. ${ }^{19}$ A skew-symmetric formulation of the convective terms in the momentum and energy 
equation is implemented for better conservation properties $\left(\right.$ e.g. $\left.{ }^{20}\right)$. The combination of SBP operators and skew-symmetric convective terms produces a robust solver that minimizes grid-point to grid-point energy build-up, and reduces the resulting numerical instabilities. A very minimal amount of explicit filtering is applied via the eighth-order Visbal and Gaidonte filter, ${ }^{21}$

$$
\alpha_{f}\left(\hat{f}_{i-1}+\hat{f}_{i+1}\right)=\frac{1}{2} \sum_{n=0}^{4} a_{N}\left(f_{i+n}+f_{i-n}\right)
$$

with $\mathrm{N}=4$ and $\alpha_{f}=0.49$, whose transfer function has a sharp cut-off at high frequencies. A Fourier spectral method is used in the spanwise direction, and the time stepping is accomplished with a forth-order Runge Kutta scheme.

\section{II.C. Simulation Details}

The grid and computational domain used for the LES simulations is given in figure 2 with every sixth grid point displayed. Current computations have 800 points in the streamwise direction, 160 in the wall normal direction and 64 points in the spanwise direction for a total of approximately 8.2 million points. The resolution at the point $x / c=-0.5$ on the wall is $\Delta x / c=0.0094, \Delta y / c=0.00087$, and $\Delta z / c=0.0031$ in the streamwise, wall normal and spanwise directions, respectively, and a typical timestep is $\Delta t a_{\infty} / c=0.00035$.

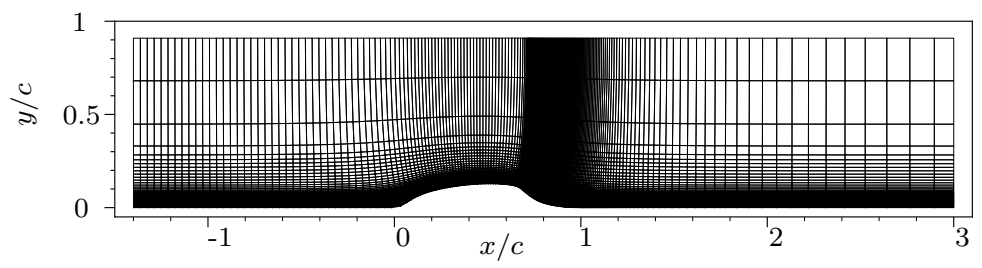

Figure 2. The computational grid (every sixth grid point plotted).

The domain size is $4.9 c \times 0.909 c \times 0.2 c$, which matches the experiments at LRCW but is shorter than the experimental domain in the spanwise and streamwise directions in order to reduce the computational cost. The height of the hump is approximately $0.12 c$ at its maximum. The Reynolds number of the simulations is 500,000 based on the chord and freestream velocity. The flow is initialized with a potential flow solution superimposed with a turbulent boundary layer profile on the lower wall. Velocity perturbations, formulated with sums of random Fourier modes, are added in a Gaussian region close to the inlet to acceleration the development of a turbulent boundary layer.

The boundary conditions are periodic in the spanwise direction, no-slip and iso-thermal conditions on the lower wall boundary, and symmetry is imposed on the upper boundary. The inflow and exit boundaries have non-reflecting boundary conditions with a buffer zone that relaxes the flow towards the initial solution. ${ }^{22}$

Rather than model the flow field inside the actuation cavity of the experiment, the boundary conditions are modified at the wall to simulate the slot jet. ${ }^{23,24}$ When actuation is applied, a normal velocity distribution is prescribed on the boundary nodes to approximate the same slot location and approximate slot width, $h_{s}$, as used in the experiments. The normal velocity at the wall is given by the Gaussian profile

$$
u_{s}=u_{s, \max } e^{-\left(x-x_{s}\right)^{2} / 2 \sigma^{2}}, \quad h_{s}=4 \sigma .
$$

The normal velocity in Eq. (8) is actuated in time by $\sin (\omega t)$ such that the mean slot velocity is zero. The non-dimensional parameters for the oscillatory control are defined by the unsteady momentum flux coefficient

$$
\left\langle C_{\mu}\right\rangle=\frac{\rho_{s}\left\langle u_{s}\right\rangle^{2} h_{s}}{0.5 \rho_{\infty} U_{\infty}^{2} c}
$$

and the reduced forcing frequency $F^{+}$in which $X_{\text {sep }}=c / 2$.

The controlled flow is started from the fully developed baseline flow. ${ }^{13}$ A range of actuation frequencies are investigated for the $M=0.6$ with a constant momentum coefficient of $C_{\mu}=0.11 \%$. The frequencies are multiple of the LRCW test case and are at $F^{+}=0.84,1.7,2.5,3.4,5.0$, and 8.4. At a lower Mach number of 
$M=0.25$, control is applied with two actuation frequencies of $F^{+}=0.84$ and 11.8. Additional cases which individually vary the momentum coefficient and the actuation location at $F^{+}=8.4$ are also investigated in the $M=0.25$ flow.

\section{Results}

\section{III.A. Controlled Mean Flow}

The baseline flow over the wall-mounted hump is characterized by boundary layer separation at $65 \%$ of the chord, followed by an unsteady turbulent recirculation region over the trailing edge. The time and spanaveraged pressure coefficient at $M=0.25$ and $M=0.6$ are displayed in figure 3 and are validated against the experimental data from Seifert and Pack $^{15}$ and Greenblatt et al. ${ }^{10}$ Pressure recovery and reattachment in the $M=0.6$ flow occurs further downstream due to the lower growth rate and entrainment rate of the separated shear layer. A more detailed study of the baseline flow can be found in Franck and Colonius. ${ }^{13}$

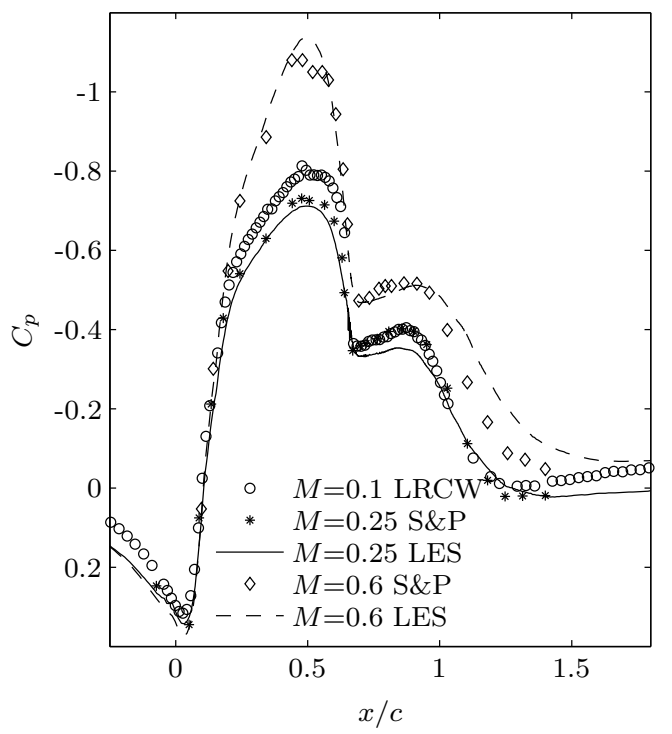

Figure 3. Pressure coefficients of $M=0.25$ vs. high $M=0.6$ baseline flow.

The average pressure coefficients for a range of actuation frequencies are shown in figure 4(a) performed at $M=0.6$. The low frequency $(\mathrm{LF})$ actuation at $F^{+}=0.84$ and 1.7 both initiate an earlier pressure recovery and reattachment. The high frequency (HF) actuation at $F^{+}=5.0,8.4$, and 11.8 slightly delay the pressure recovery and reattachment compared to the baseline flow. The two frequencies in between the $\mathrm{LF}$ and $\mathrm{HF}, F^{+}=2.5$ and 3.4, do not have a significant effect on the average $C_{p}$, and are very close to the average baseline flow. At a lower Mach number of 0.25 , two actuation frequencies are investigated, a high frequency of $F^{+}=11.8$ and a low frequency of $F^{+}=0.84$, whose averaged pressure coefficients are given by figure $4(\mathrm{~b})$. The $M=0.25 \mathrm{LF}$ actuation results in a shorter recirculation region as seen by the earlier pressure recovery, similar to that seen in the $M=0.6$ flow. The $M=0.25 \mathrm{HF}$ actuation is very similar to the average baseline flow, with no major change in the reattachment location.

The average pressure coefficients in figure 4 are all from cases performed with a momentum coefficient of $\left\langle C_{\mu}\right\rangle=0.11 \%$. Figure 5 displays the pressure coefficient of two HF cases at a higher momentum coefficient of $\left\langle C_{\mu}\right\rangle=0.23 \%$. The controlled case in figure $5(\mathrm{~b})$ is actuated at $x / c=0.655$, the same location as the cases in figure 4 , which also corresponds with the location of previously performed experiments. ${ }^{10}$ This actuation location is immediately upstream of the mean, baseline separation point, which is optimal in the low frequency and low Mach experiments of Seifert and Pack. ${ }^{15}$ To determine if the HF actuation is sensitive to the actuation location, one case is actuated further upstream at $x / c=0.60$, with $\left\langle C_{\mu}\right\rangle=0.23 \%$. As seen figure 4, modifying the actuation location and the momentum coefficient of the high frequency actuation did not have a significant effect on the mean separation and reattachment behavior. There is a small increase in pressure at the actuation location, which is more distinguishable when the flow is actuated at the $x / c=0.60$ location, but it does not have any global effects on the mean flow. 


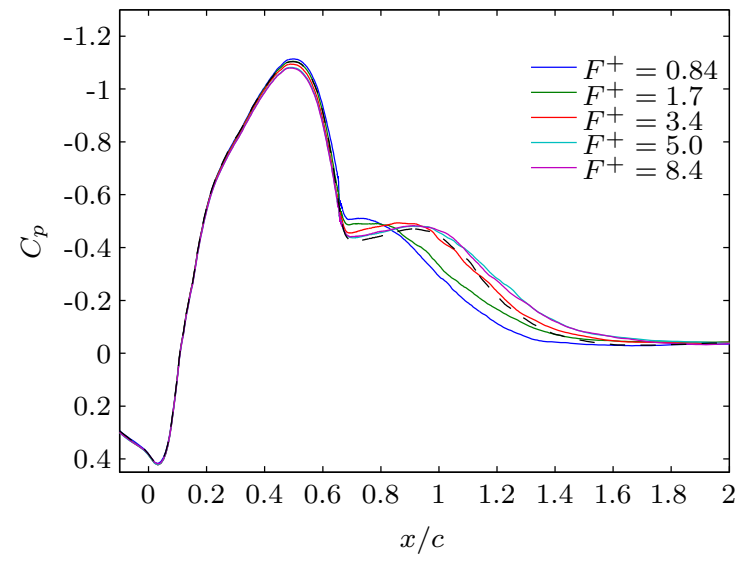

(a) $M=0.6$

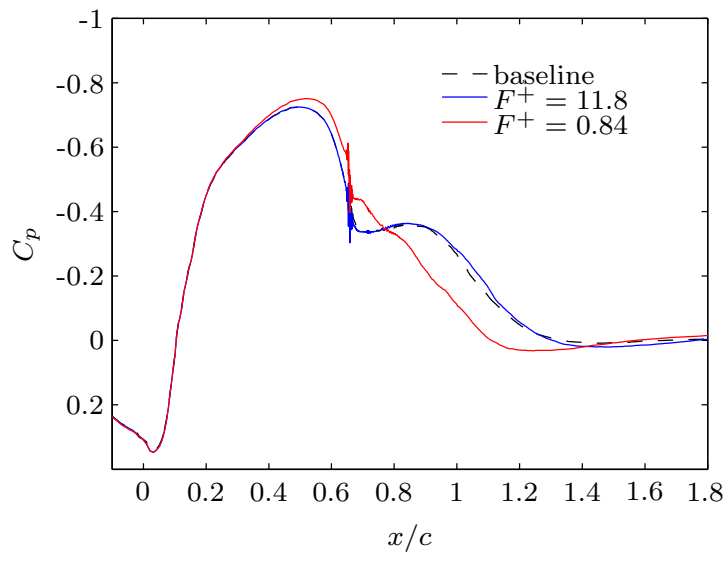

(b) $M=0.25$

Figure 4. The effect of actuation frequency on the average pressure coefficient. For all actuation frequencies: $\left\langle C_{\mu}\right\rangle=$ $0.11 \%$

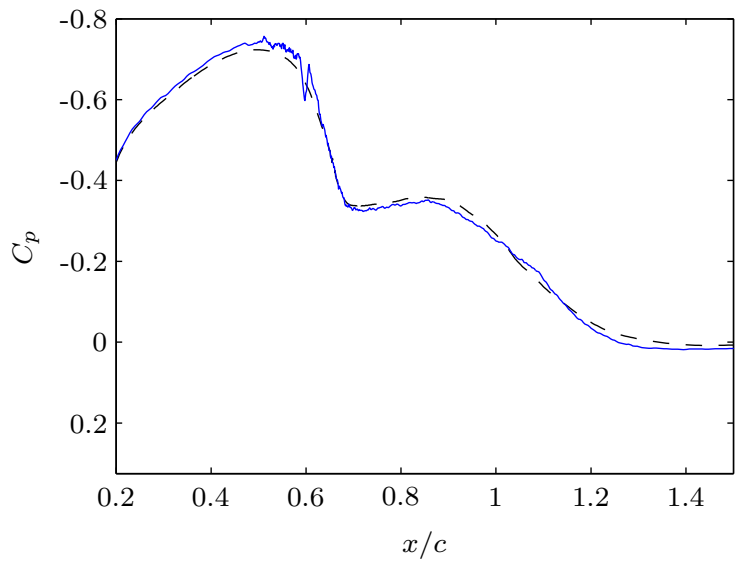

(a) Actuation location

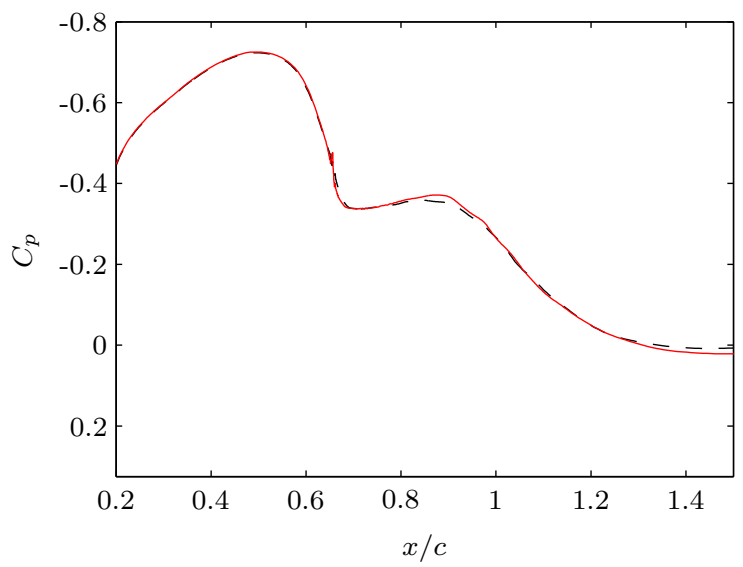

(b) Actuation strength

Figure 5. The effect of location and $C_{\mu}$ of high frequency actuation on the average pressure coefficient. Left figure: baseline (dashed) and $F^{+}=11.8,\left\langle C_{\mu}\right\rangle=0.23 \%$ actuated at $x / c=0.60$ (blue). Right figure: baseline (dashed) and $F^{+}=11.8,\left\langle C_{\mu}\right\rangle=0.23 \%$ actuated at $x / c=0.655$ (red). 
A similar trend is seen in the average velocity contours in figure 6 where the $M=0.6$ low and high frequency actuation are compared with the baseline flow. The $u / U \infty$ contours display a shorter recirculation region with LF actuation, but the HF actuated velocity contours appear similar to the baseline flow. The LF shows an increase in the reverse flow beneath the shear layer, and also an increase in negative vertical velocity above the shear layer. The increase in velocity magnitude indicates a higher rate of fluid entrainment into the shear layer from both the high speed fluid above, and the low speed fluid below the shear layer. The increased entrainment rate caused by LF actuation accelerates the reattachment of the shear layer, and shortens the mean separated region. Conversely, the HF actuation case has lower magnitudes of vertical velocity above the shear layer than the baseline flow. This could be due to lower levels of entrainment from the freestream fluid, or a net deflection of the shear layer away from the wall.
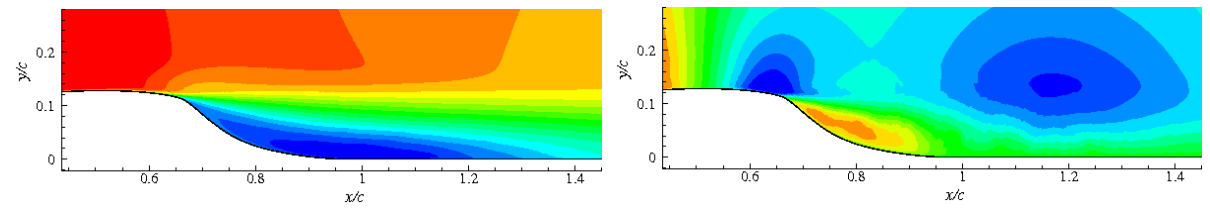

(a) $M=0.6$ baseline
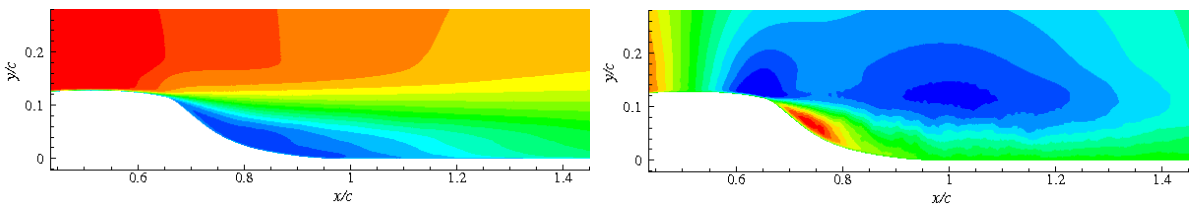

(b) $M=0.6 F^{+}=0.84$
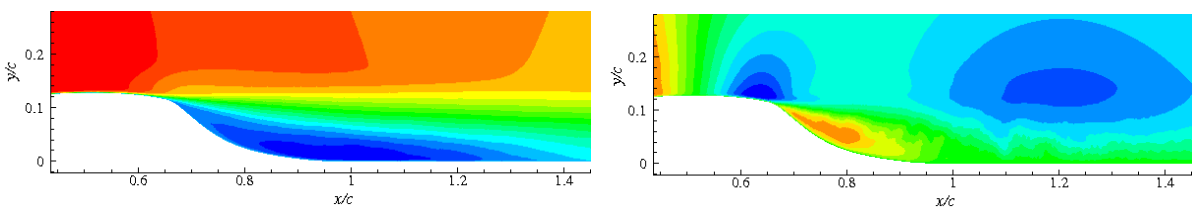

(c) $M=0.6 F^{+}=8.4$

Figure 6. Average velocities $\bar{u} / U_{\infty}$ (left) and $\bar{v} / U_{\infty}$ (right). $\bar{u} / U_{\infty}$ contour levels from -0.2 to $1.3, \bar{v} / U_{\infty}$ contour levels from -0.1 to 0.1 .

\section{III.B. Local Effects of Actuation}

To look at the local effect of actuation, the momentum and vorticity thickness are calculated. The momentum thickness, as defined below, is modified to adjust for the acceleration of the freestream flow,

$$
\theta(x)=\int_{y_{\min }}^{\infty} \frac{\bar{u}(x, y)-u_{\min }(x)}{u_{\max }(x)-u_{\min }(x)}\left(1-\frac{\bar{u}(x, y)-u_{\min }(x)}{u_{\max }(x)-u_{\min }(x)}\right),
$$

and the vorticity thickness is defined by

$$
\delta_{\omega}(x)=1 /\left|\frac{d\left(u(x) / U_{\infty}\right)}{d y}\right|_{\max } .
$$

The local momentum thickness surrounding actuation is plotted for the baseline and controlled cases in figure 7. The LF actuation has an increase in the momentum thickness of $24 \%$ at $M=0.25$ compared to baseline, and a more moderately increase of $12 \%$ for the $M=0.6$ flow. The modification in momentum thickness is both upstream and downstream of the actuation location. As the actuation frequency is increased, the momentum thickness decreases, resulting in the smallest value at the highest actuation frequencies. The two highest actuation frequencies have the same momentum thickness, which is less than the baseline. It also implies that there is a threshold frequency above which the boundary layer will have the same response. The HF actuation reduces the momentum thickness by approximately $10 \%$ at $M=0.6$ and $6 \%$ at $M=0.25$.

The spatial growth of the vorticity thickness is calculated with a linear fit beginning just after separation at $x / c=0.67$. The growth rate of the shear layer is not linear, but the initially separated region is 


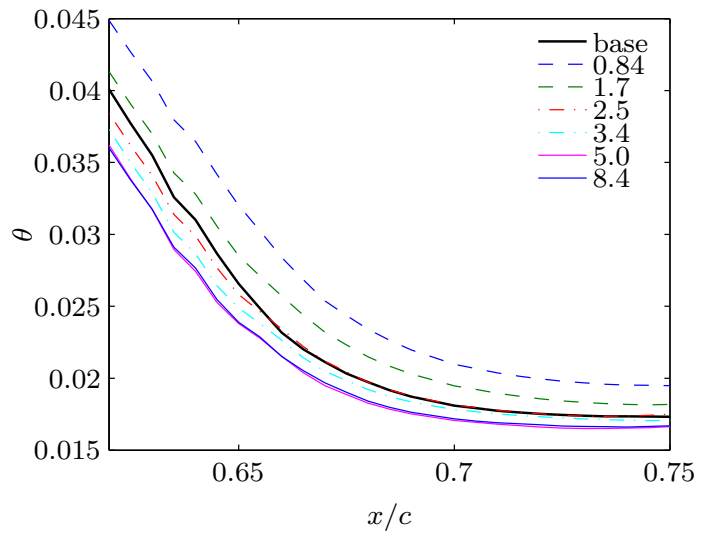

(a) $M=0.6$

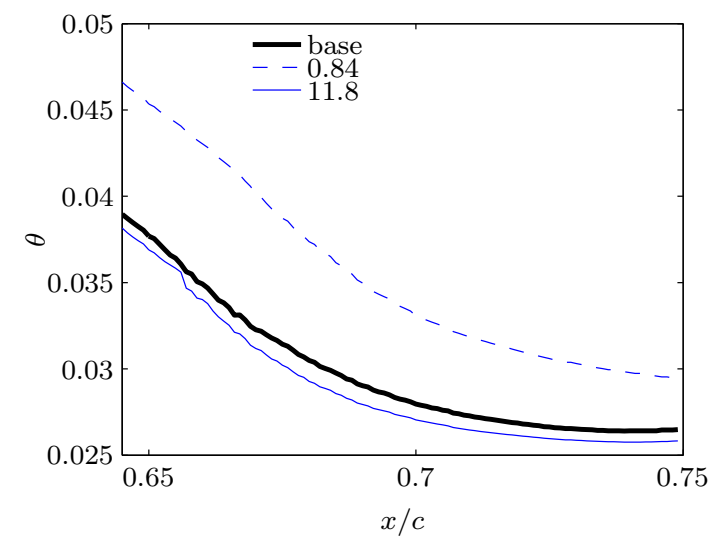

(b) $M=0.25$

Figure 7. Boundary layer momentum thickness with various forcing frequencies. Control is applied at $x / c=0.655$.

approximated by a linear fit to obtain a measure of the shear layer's growth rate. Estimates of the initial $d \delta_{\omega} / d(x / c)$ are given in table 1 for the controlled $M=0.25$ and $M=0.6$ flows. The $M=0.25$ results indicate a decrease in $d \delta_{\omega} / d(x / c)$ for HF actuation and an increase for LF actuation compared with the baseline flow. At $M=0.6, d \delta_{\omega} / d(x / c)$ decreases with increasing frequency, and is slightly less than the baseline growth rate at the highest frequency. Thus, LF actuation is actively increasing the growth of the shear layer whereas HF actuation slightly diminishes the growth rate immediately after separation. The Reynolds stress profiles at $x / c=0.67$ are plotted in figure 8. With HF actuation, the Reynolds stresses are lower than the baseline flow, whereas they are higher than baseline when LF actuation is applied. The lower level of velocity fluctuations with HF actuation may be responsible for the initially lower growth and entrainment rates.

Table 1. Initial growth rate of controlled flow from a linear
fit of $d \delta_{\omega} / d(x / c)$ between $0.67<x / c<0.76(M=0.6)$ and
$0.67<x / c<0.70(M=0.25)$.
\begin{tabular}{ccc} 
Mach & case & $d \delta_{\omega} / d(x / c)$ \\
\hline 0.6 & baseline & 0.231 \\
0.6 & $F^{+}=0.84$ & 0.285 \\
0.6 & $F^{+}=1.7$ & 0.244 \\
0.6 & $F^{+}=2.5$ & 0.245 \\
0.6 & $F^{+}=3.5$ & 0.231 \\
0.6 & $F^{+}=5.0$ & 0.231 \\
0.6 & $F^{+}=8.4$ & 0.223 \\
\hline 0.25 & baseline & 0.313 \\
0.25 & $F^{+}=0.84$ & 0.465 \\
0.25 & $F^{+}=11.8$ & 0.283 \\
\hline
\end{tabular}

The averaged streamlines surrounding high frequency actuation are shown in figure 9 and compared with the baseline case. When HF actuation is applied, the local streamlines are deflected towards the wall, an effect that is seen more clearly with increasing momentum coefficient, as seen in figures $9(\mathrm{e})$ and $9(\mathrm{f})$. This deflection corresponds to a very slight modification in the local streamwise pressure coefficient in figure 5 (b), but does not significantly effect the pressure downstream or upstream of the actuation. Despite the streamline deflection closer to the wall, the average streamlines in figure 9 indicate that the flow separates at the same location as in the baseline flow. After separation, the HF actuation produces a slightly wider reverse flow region, which may deflect the mean shear layer further away from the wall. When the flow is actuated further upstream of separation at $x / c=0.6$, the local pressure coefficient is modified, as seen in figure $5(\mathrm{a})$, but has a very minimal effect on the local streamlines in $9(\mathrm{~g})$. 
(a) $M=0.6$
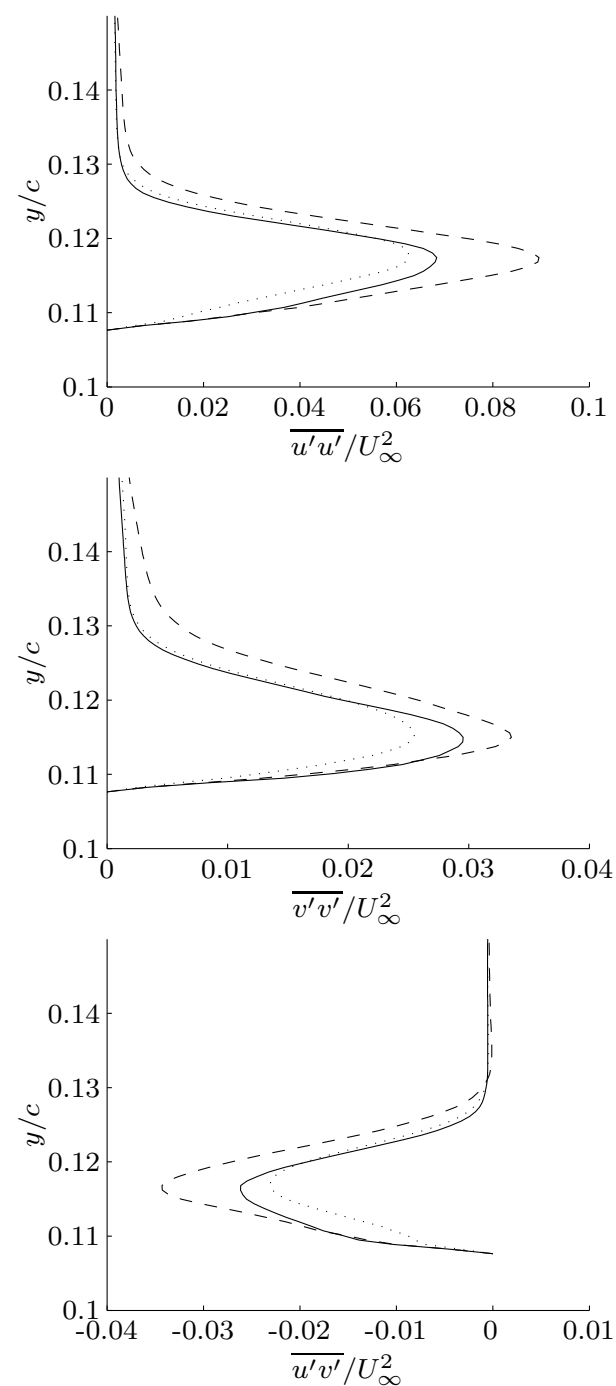

(b) $M=0.25$
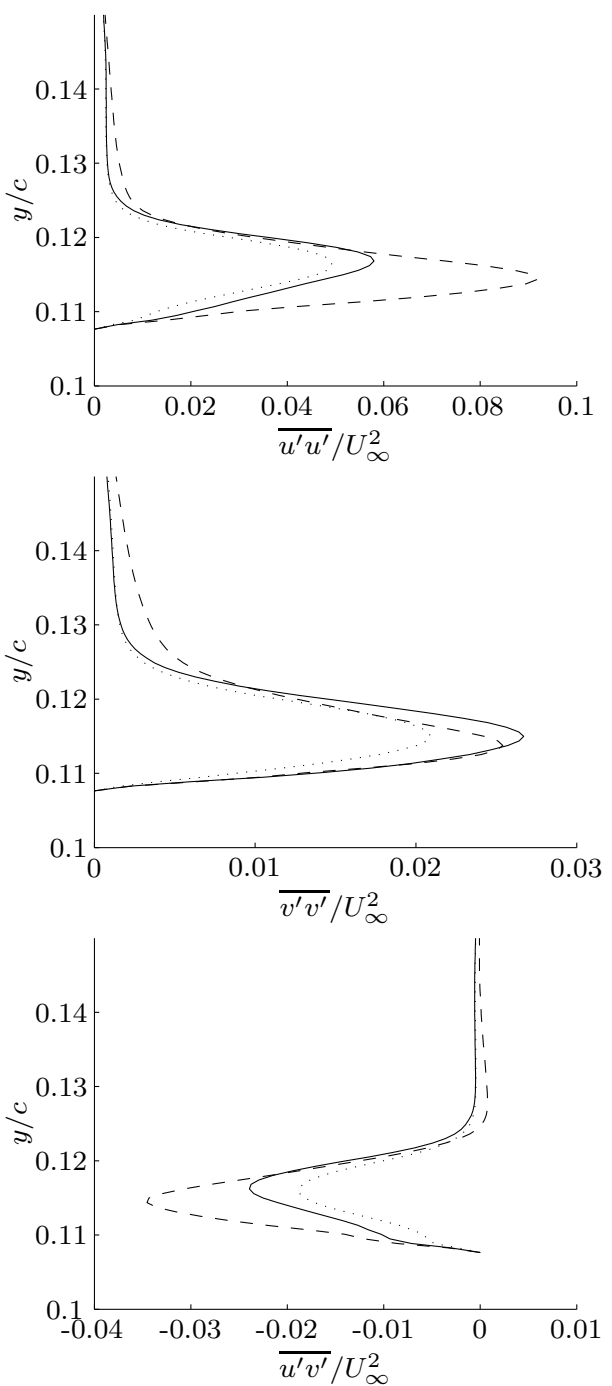

Figure 8. Resolved turbulent Reynolds stresses at $x / c=0.67$ for: baseline flow (solid line), LF actuation (dashed), and HF actuation (dotted). 


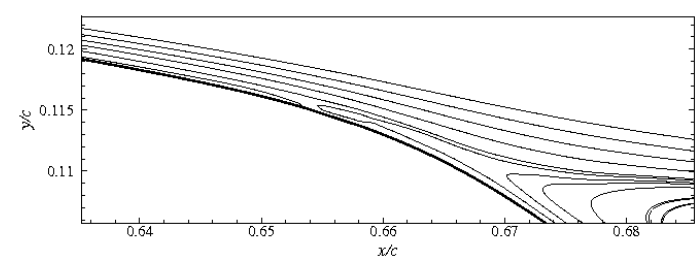

(a) $M=0.6$ : baseline

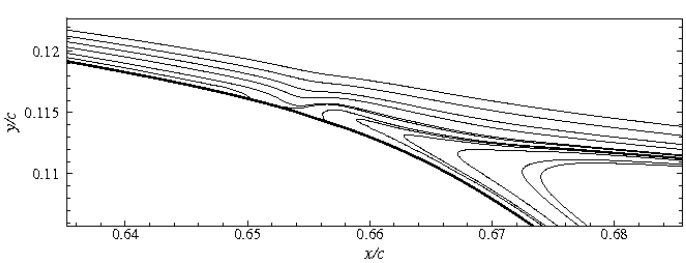

(c) $M=0.6: F^{+}=8.4,\left\langle C_{\mu}\right\rangle=0.11 \%$

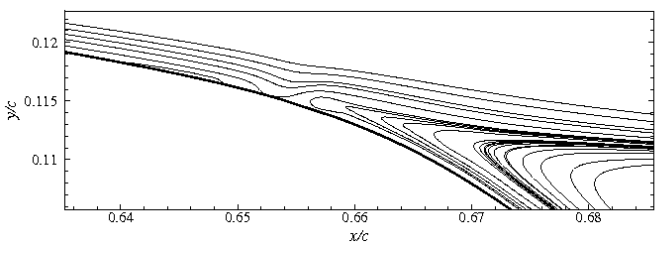

(e) $M=0.6: F^{+}=8.4,\left\langle C_{\mu}\right\rangle=0.16 \%$

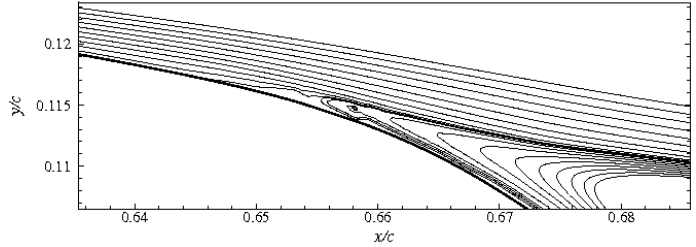

(b) $M=0.25$ : baseline

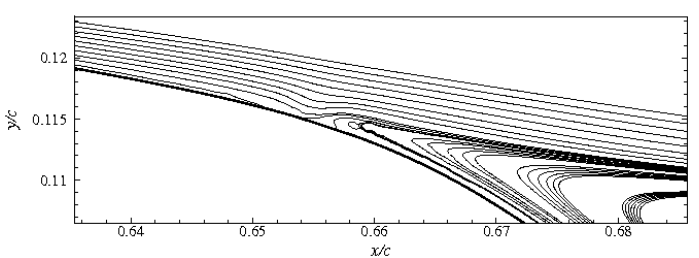

(d) $M=0.25: F^{+}=11.8,\left\langle C_{\mu}\right\rangle=0.11 \%$

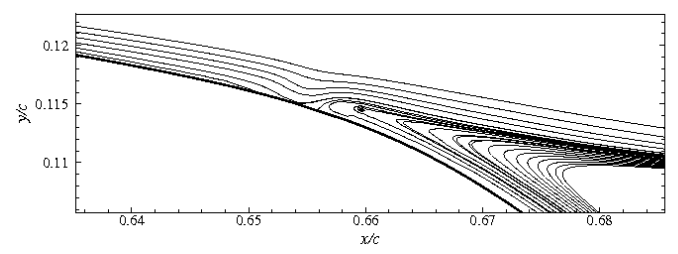

(f) $M=0.25: F^{+}=11.8,\left\langle C_{\mu}\right\rangle=0.23 \%$

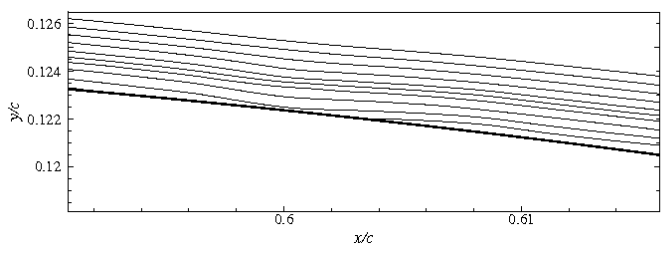

(g) $M=0.25: F^{+}=11.8,\left\langle C_{\mu}\right\rangle=0.23 \%$

Figure 9. Averaged streamlines surrounding separation and actuation. Actuation is centered at $x / c=0.655$ for (c) - (f) and at $x / c=0.60$ for $(\mathrm{g})$. 


\section{III.C. Unsteady Effects of Actuation}

The phase-averaged spanwise vorticity of three actuation frequencies, $F^{+}=0.84,1.7$, and 5.0, are shown in figure 10. With LF actuation, coherent structures form at separation and are shed from the shear layer as depicted in the LF suction and actuation phases in figures 10(a)-10(d). As the frequency increases, structures that result from actuation are no longer distinguishable in the phase-averaged vorticity. At $M=0.6$ and $F^{+} \geq 5.0$ there is no difference between the suction and blowing phase-averaged vorticity contours in figures $10(\mathrm{e})$ and $10(\mathrm{f})$.

Velocity probes of the span-averaged flow are placed within the shear layer and along the wall within the separated region. The spectra in figures 11 represent probes at various locations along the separated region, including a probe at the actuation location itself. The LF and HF actuation cases are directly compared to the corresponding baseline spectra.

At $M=0.6$, the LF actuated flow displays strong spectral peaks at the forcing frequency for all locations within the shear layer. The most distinct peaks at the actuation frequency occur at actuation $(x / c=0.655)$ and just after separation $(x / c=0.67)$. In the downstream portion of the separation bubble, frequencies lower than the actuation frequency grow in strength indicating that vortices may merge as the separation bubble reattaches. However, the lack of a distinct peak at the subharmonic of the actuation frequency indicates that pairing is not regular. The growth of lower frequencies decreases the actuation frequency peak at $x / c=0.86$, but at reattachment $(x / c=1.1)$ the actuation frequency has increased again. Pressure probes at the wall also contain peaks at the actuation frequency, but the pressure-spectra peaks are lower than the velocity-spectra peaks from the shear layer probe. In addition, the amplitude of the actuation peaks in the pressure spectra decrease significantly close to reattachment $(x / c=1.1)$.

When the $M=0.6$ flow is actuated at $F^{+}=5$, the velocity spectra displays a peak at the actuation frequency directly at the actuation location of $x / c=0.655$. This peak is also detectable immediately after separation at $x / c=0.69$, along with a subharmonic at $F^{+}=2.5$. However the actuation frequency is no longer present towards the middle of the separation bubble $(x / c=0.85)$ and close to reattachment $(x / c=1.1)$. This is consistent with the lack of coherent structures in the phase-averaged vorticity contours in figure 10. This is contrary to the LF actuation, which maintains a peak at the actuation frequency at least until reattachment. With HF actuation, the associated flow structures are too small, and dissipate too quickly to be separated from the turbulence in the separated region. Thus, they have a very minor effect on the reorganization of the flow. Despite the weak appearance of the actuation in the velocity spectra, the pressure spectra have detectable peaks at the actuation frequency for all probes. If one looks at the pressure contours over time, there is a strong acoustic disturbance that emanates from the actuation location. Therefore, although the actuation may not significantly affect the mean flow, it does have a strong effect on the acoustic field.

\section{Discussion and Summary}

Low frequency actuation at $F^{+} \sim O(1)$ was compared to higher frequency actuation at $F^{+} \geq 5$ for Mach numbers 0.6 and 0.25 using an LES code previously validated for the wall-mounted hump flow. ${ }^{13,14}$ Actuation at $F^{+} \sim O(1)$ has been shown experimentally to create regular, large scale structures that increase the entrainment into the separated shear layer, and reduce the average separation length. ${ }^{9,11}$ Utilizing a LES of the wall-mounted hump flow, the mean, local, and unsteady effects of high frequency actuation were investigated and compared to the effects of low frequency actuation.

In this investigation, high frequency actuation applied to the wall-mounted hump geometry does not improve the overall drag, nor reduce the overall separation bubble length. At higher frequencies the vorticity structures generated at actuation were small and dissipated very rapidly. At $M=0.6$, the shear layer reattached slightly downstream of the baseline flow, and at $M=0.25$ reattachment was approximately equal to that of the baseline flow. The pressure coefficient at separation and within the separation bubble is mostly unchanged. This is contrary to the experimental results of Amitay and Glezer ${ }^{1}$ who found that HF excitation just before separation on the suction side of a stalled airfoil was beneficial to the aerodynamic performance, by increasing the suction peak, and seemingly reattaching the boundary layer. However, other investigations have also reported an increase in separation bubble length with $\mathrm{HF}$ actuation, ${ }^{4,5}$ and a decrease in separation length when LF actuation was applied. Raju et al. found a mild enhancement of separation when HF actuation is applied to the 2D simulations around an airfoil at high angle-of-attack. ${ }^{4}$ In the LES of a curved backward-facing step, Dandois et al. ${ }^{5}$ found a reduction in separation bubble length of $54 \%$ with LF 


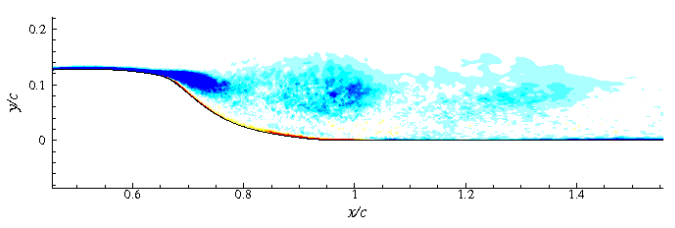

(a) $F^{+}=0.84:$ phase $=90^{\circ}$

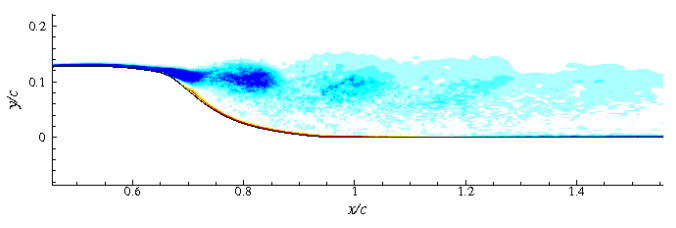

(c) $F^{+}=1.7:$ phase $=90^{\circ}$

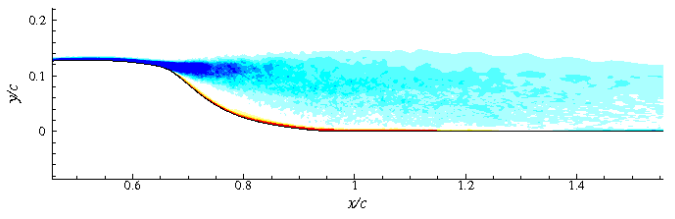

(e) $F^{+}=5.0:$ phase $=90^{\circ}$

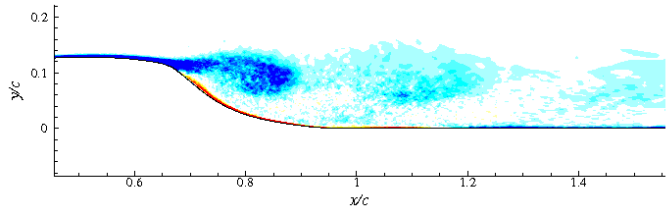

(b) $F^{+}=0.84:$ phase $=270^{\circ}$

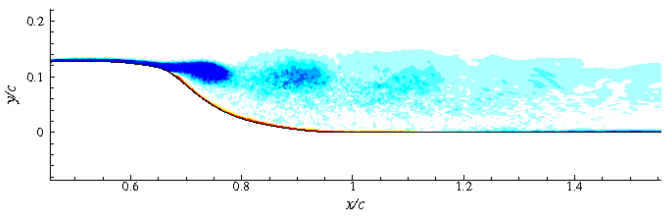

(d) $F^{+}=1.7:$ phase $=270^{\circ}$

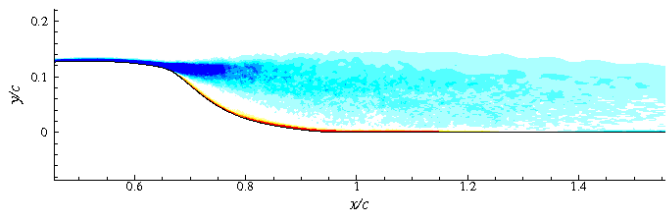

(f) $F^{+}=5.0:$ phase $=270^{\circ}$

Figure 10. Phase-averaged spanwise vorticity corresponding to the blowing $\left(\mathrm{phase}=90^{\circ}\right)$ and suction $\left(\mathrm{phase}=270^{\circ}\right)$ phases. Contour levels from -30 to 30 .

actuation, and an increase in $43 \%$ when HF actuation was applied. However, the computations of Dandois et al. had a relatively high momentum coefficient of $\left\langle C_{\mu}\right\rangle=1 \%$ compared to experimental investigations.

The mean flow can also be examined locally at the actuation location. Previous investigations have hypothesized that HF actuation can modify the mean streamwise velocity profile, increasing stability of the boundary layer and inhibiting growth of large scale structures. ${ }^{25}$ If the HF actuation were successful in inhibiting large-scale structure growth, it would for example be beneficial in reducing acoustic resonance generated by these structures. ${ }^{25}$ The LES of Dandois et al. also found that the high frequency forcing inhibits the growth of large scale structures by creating a more stable average velocity profile. ${ }^{5}$ Other investigators have found that HF actuation can locally delay separation by stabilizing the local boundary layer and modifying the streamwise pressure gradient. ${ }^{2}$ With the wall-mounted hump, HF actuation does modify the local streamlines surrounding actuation, but does not delay separation or modify the stability of the boundary layer. At $M=0.25$ when the momentum coefficient is increased to $23 \%$, there is a modification in the streamwise pressure profile, but it is only local and does not significantly affect the pressure within the separation bubble and at reattachment.

High frequency actuation does lower the local levels of velocity fluctuations, a trend also seen surrounding the actuators in the airfoil experiments by Amitay and Glezer, ${ }^{1}$ especially in the cross-stream fluctuations $\overline{v^{\prime} v^{\prime}}$. In these experiments, the lowered fluctuations increase the stability of the boundary layer, resulting in separation delay and increased suction. However, in the current experiments the lower levels of fluctuations are likely responsible for the lower growth rate in the vicinity of actuation. The lower level of velocity fluctuations is also associated with a higher degree of boundary layer deflection from the surface when compared to the baseline flow.

Amitay et al. also note that the addition of high frequency actuation decreases the separated flow region without the high aerodynamic fluctuations that low frequency actuation imposes. ${ }^{1}$ The low aerodynamic fluctuations are due to the fact that the high frequency actuation does not create large vortices, but very small ones that are dissipated quickly. Likewise Dandois et al. ${ }^{5}$ have called the low frequency actuation a "vorticity dominated mode", where large scale vortices are created, and the high frequency actuation a "acoustic dominated mode", due to the acoustic noise that evolves from the actuation location. A spectral analysis of the wall-mounted hump flow also shows stronger peaks at the actuation frequency in the pressure spectra, which may be the result of acoustic disturbances from the actuation. However the velocity spectra only has weak peaks at the actuation frequency, indicating weaker vortical structures in contrast to the 


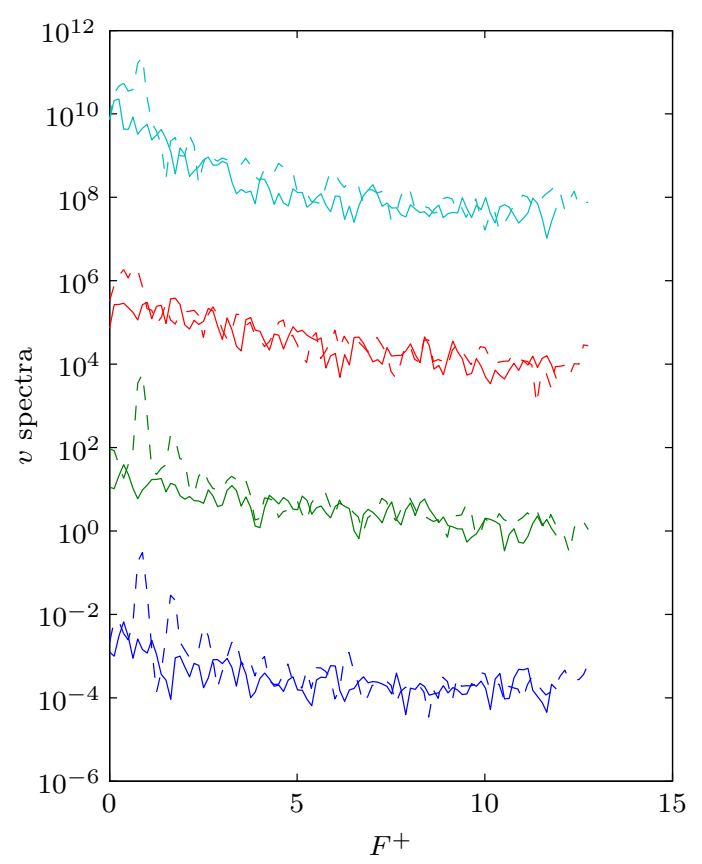

(a) $\mathrm{M}=0.6 \mathrm{LF}$ : $v$ spectra in the shear layer

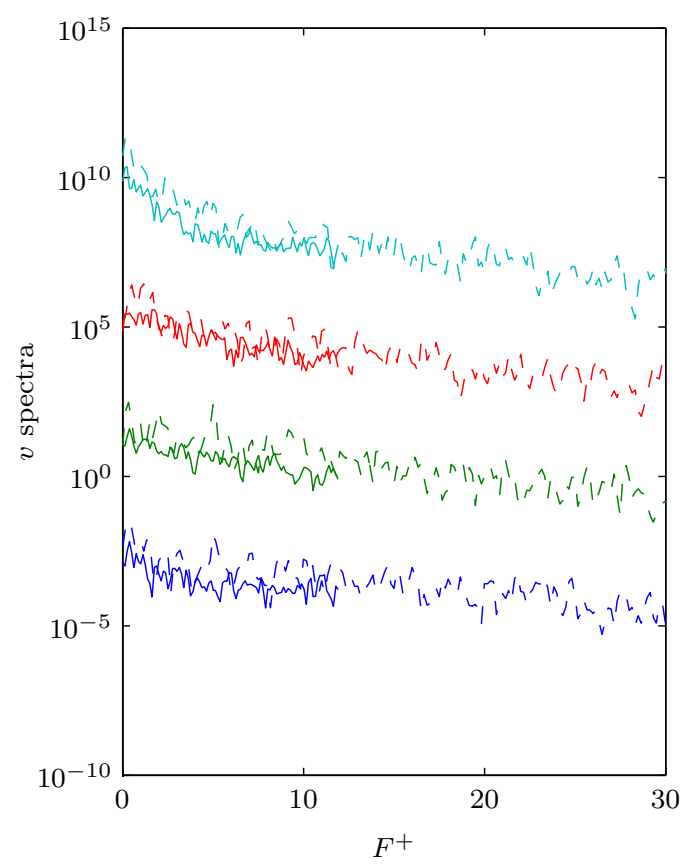

(c) $\mathrm{M}=0.6 \mathrm{HF} v$ spectra in the shear layer

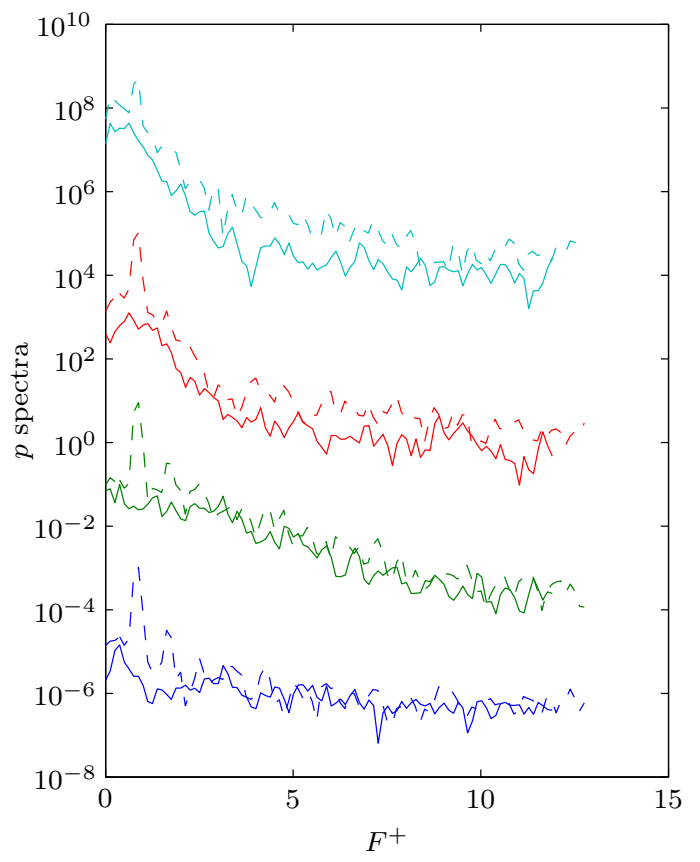

(b) $\mathrm{M}=0.6 \mathrm{LF}: p$ spectra at the wall

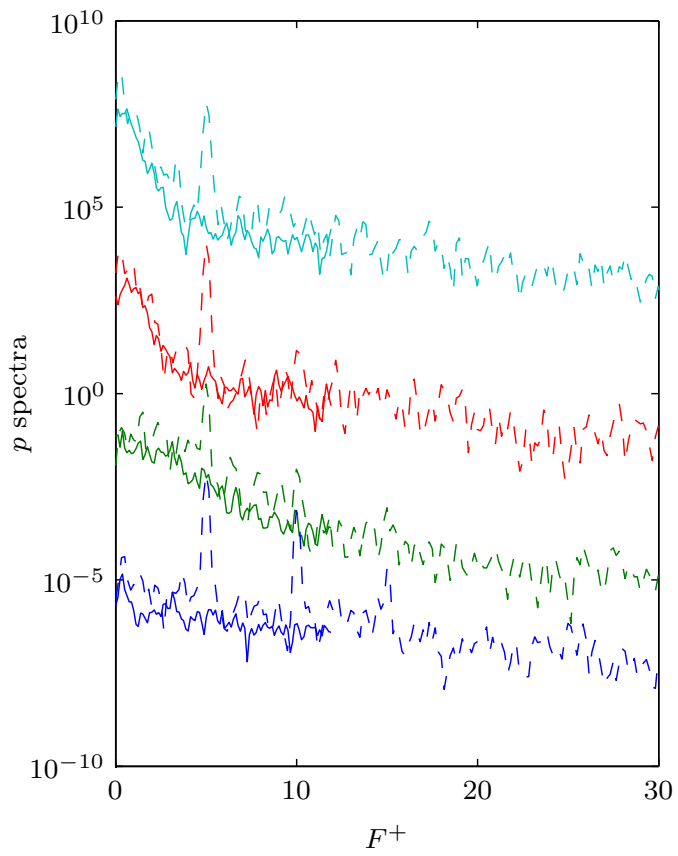

(d) $\mathrm{M}=0.6 \mathrm{HF}: p$ spectra at the wall

Figure 11. $M=0.6$ spectra from the span-averaged flow at four locations, separated by three decades for easier viewing. Locations are $x / c=0.655$ (blue), 0.69 (green), 0.86 (red), and 1.1 (cyan), baseline (solid), controlled (dashed). 
strong vortices created by low frequency actuation.

The low frequency actuation is successful in reducing the separation bubble size by the creation of large vortical structures which increase the momentum thickness and growth of the separated shear layer. The larger averaged vorticity thickness promotes entrainment intro the shear layer and results in an earlier reattachment. However, with the same location and $C_{\mu}$ values, high frequency actuation decreases the local velocity fluctuations and slightly lowers the growth rate of the vorticity thickness below that of the baseline flow. The resulting mean flow is very similar to the baseline flow, with a slightly larger separation bubble size found when high frequency actuation is applied to the $M=0.6$ flow. Thus, high frequency actuation was not found to be beneficial for drag reduction or shortening the separation bubble on a wall-mounted hump geometry.

\section{Acknowledgements}

This work was supported by a National Science Foundation Graduate Student Fellowship and the US Air Force Office of Scientific Research grant FA9550-05-1-0369.

\section{References}

${ }^{1}$ Amitay, M. and Glezer, A., "Role of Actuation Frequency in Controlled Flow Reattachment over Stalled Airfoil," AIAA Journal, Vol. 40, No. 2, 2002, pp. 209-216.

${ }^{2}$ Glezer, A., Amitay, M., and Honohan, A., "Aspects of low- and high-frequency actuation for aerodynamic flow control," AIAA Journal, Vol. 43, No. 7, Jul 2005, pp. 1501-1511.

${ }^{3} \mathrm{Wu}$, J., Lu, X., Denny, A., Fan, M., and Wu, J., "Post-stall flow control on an airfoil by local unsteady forcing," Journal of Fluid Mechanics, Vol. 371, Sep 1998, pp. 21-58.

${ }^{4}$ Raju, R., Mittal, R., and Cattafesta, L., "Dynamics of Airfoil Separation Control Using Zero-Net Mass-Flux Forcing," AIAA Journal, Vol. 46, No. 12, Dec 2008, pp. 3103-3115.

${ }^{5}$ Dandois, J., Garnier, E., and Sagaut, P., "Numerical simulation of active separation control by a synthetic jet," Journal of Fluid Mechanics, Vol. 574, Mar 10 2007, pp. 25-58.

${ }^{6}$ Nishri, B. and Wygnanski, I., "Effects of Periodic Excitation on Turbulent Fow Separation from a flap." AIAA Journal, Vol. 36, No. 4, 1998 , pp. 547-556.

${ }^{7}$ Seifert, A. and Pack, L., "Active Control on Generic Configurations at High Reynolds Numbers," 30th AIAA Fluid Dynamics Conference, Jun 1999.

${ }^{8}$ Greenblatt, D. and Wygnanski, I. J., "The control of flow separation by periodic excitation," Progress in Aerospace Sciences, Vol. 36, 2000, pp. 487-545.

${ }^{9}$ Seifert, A. and Pack, L., "Active flow separation control on wall-mounted hump at high Reynolds numbers," AIAA Journal, Vol. 40, No. 7, Jul 2002, pp. 1363-1372.

${ }^{10}$ Greenblatt, D., Paschal, K. B., Yao, C.-S., Harris, J., Schaeffler, N.-N. W., and Washburn, A. E., "Experimental investigation of separation control - Part 1: Baseline and steady suction," AIAA Journal, Vol. 44, No. 12, Dec 2006, pp. $2820-2830$.

${ }^{11}$ Greenblatt, D., Paschal, K. B., Yao, C.-S., and Harris, J., "Experimental investigation of separation control - Part 2: Zero mass-flux oscillatory blowing," AIAA Journal, Vol. 44, No. 12, Dec 2006, pp. 2831-2845.

${ }^{12}$ Rumsey, C. L., Gatski, T. B., Sellers, W. L., Vatsa, V. N., and Viken, S. A., "SumMary of the 2004 computational fluid dynamics validation workshop on synthetic jets," AIAA Journal, Vol. 44, No. 2, 2006, pp. 194-207.

${ }^{13}$ Franck, J. A. and Colonius, T., "A Compressible Large-Eddy Simulation of Separation Control on a Wall-Mounted Hump." 46th AIAA Aerospace Sciences Meeting and Exhibit, Jan 2008.

${ }^{14}$ Franck, J. A. and Colonius, T., "Compressible Large-Eddy Simulation of Separation Control on a Wall-Mounted Hump," AIAA Journal (submitted), 2009.

${ }^{15}$ Seifert, A. and Pack, L., "Compressibility and Excitation Location Effects on High Reynolds Numbers Active Separation Control," Journal of Aircraft, Vol. 40, No. 1, 2003, pp. 110-119.

${ }^{16}$ Moin, P., Squires, K., Cabot, W., and Lee, S., "A dynamic subgrid-scale model for compressible turbulence and scalar transport," Physics of Fluids A-Fluid Dynamics, Vol. 3, No. 11, Nov 1991, pp. 2746-2757.

${ }^{17}$ Gloerfelt, X., Bogey, C., and Bailly, C., "Numerical Evidence of Mode Switching in the Flow-Induced Oscillations by a Cavity," International Journal of Aeroacoustics, Vol. 2, No. 2, 2003, pp. 99-123.

${ }^{18}$ Driscoll, T. A. and Trefethen, L. N., Schwarz-Christoffel Mapping, Cambridge University Press, 2002.

${ }^{19}$ Mattsson, K. and Nordström, J., "Summation by parts operators for finite difference approximations of second derivatives," J. Comput. Phys., Vol. 199, No. 2, 2004, pp. 503-540.

${ }^{20}$ Honein, A. E. and Moin, P., "Higher entropy conservation and numerical stability of compressible turbulence simulations," J. Comput. Phys., Vol. 201, No. 2, 2004, pp. 531-545.

${ }^{21}$ Visbal, M. and Gaitonde, D., "High-order-accurate methods for complex unsteady subsonic flows," AIAA Journal, Vol. 37, No. 10, Oct 1999, pp. 1231-1239.

${ }^{22}$ Freund, J., "Proposed inflow/outflow boundary condition for direct computation of aerodynamic sound," AIAA Journal, Vol. 35, No. 4, Apr 1997, pp. 740-742. 
${ }^{23}$ You, D., Wang, M., and Moin, P., "Large-eddy simulation of flow over a wall-mounted hump with separation control," AIAA Journal, Vol. 44, No. 11, Nov 2006, pp. 2571-2577.

${ }^{24}$ Postl, D. and Fasel, H., "Direct numerical simulation of turbulent flow separation from a wall-mounted hump," AIAA Journal, Vol. 44, No. 2, Feb 2006, pp. 263-272.

${ }^{25}$ Stanek, M., Raman, G., Ross, J., Odedra, J., Peto, J., Alvi, F., and Kibens, V., "High Frequency Acoustic SuppressionThe Role of Mass Flow, The Notion of Superposition, And The Role of Inviscid Instability - A New Model (Part II)." 8th AIAA/CEAS Aeroacoustics Conference and Exhibit, Jun 2002. 